\title{
Participar o No PARTICIPAR: ANÁlisis TIPOlÓGICO DE LA PARTICIPACIÓN CIUDADANA DE LOS MEXICANOS
}

\author{
To Participate or Not to Participate: A Typological Analysis of \\ Mexicans' Citizen Participation
}

\section{FERNANDO NIETO}

Centro de Estudios Internacionales de El Colegio de México

FERNANDA SOMUANO

Centro de Estudios Internacionales de El Colegio de México

\begin{abstract}
RESUMEN
El déficit de participación política usualmente se identifica con la apatía y desencanto con la democracia. No obstante, poco se ha teorizado y estudiado la relación entre participación política y otras formas de involucramiento cívico, incluyendo la posibilidad de que algunos ciudadanos prefieran participar fuera o en los márgenes de la política institucional. En este trabajo, estudiamos diferentes formas de participación política y comunitaria. Partimos del supuesto de que la falta de participación en política no necesariamente implica falta de participación ciudadana. Con base en datos de encuesta, identificamos distintos tipos en función de patrones empíricos de participación en México. El análisis tipológico de conglomerados de k medias demuestra que se pueden identificar al menos cuatro tipos de participación: convencional, apático, apolítico e hiperactivo. Mediante un modelo multinomial examinamos las bases de esta tipología.
\end{abstract}

Palabras clave: Participación política, apatía, participación comunitaria, democracia, México.

\begin{abstract}
The lack of political participation is usually identified with apathy and disenchantment with democracy. However, little has been theorized and studied about the relationship between political participation and other forms of civic involvement, including the possibility that some citizens prefer to participate outside of or at the margins of institutional politics. In this study, we analyze different forms of political and community participation. We begin with the assumption that lack of participation in politics does not necessarily imply lack of citizen's participation altogether. Based on survey data, we identify types based on different empirical patterns of participation in Mexico. The typological k-means analysis shows that at least four types of participation can be identified: conventional, apathetic, apolitical and hyperactive. Through a multinomial model we examine the bases of this typology.
\end{abstract}

Keywords: Political participation, apathy, community participation, democracy, Mexico. 


\section{INTRODUCCIÓN}

La participación ciudadana es central para la gobernabilidad y vida democráticas (Marshall 1950; Isin y Wood 1999; Heywood 2000). Esta afirmación ha motivado investigación sobre los perfiles sociales y políticos de quienes participan en política y sobre las características y prevalencia de diferentes tipos de participación (Leighley 1995; Norris 2002; Dalton 2008). Estos estudios han producido una cantidad considerable de observaciones sobre patrones de participación política en diferentes sociedades - principalmente en democracias desarrolladas-, contribuyendo así a nuestra capacidad para comprender los determinantes y la frecuencia de la participación, especialmente en lo que respecta al comportamiento electoral (por ejemplo, Norris 2002; Kam 2012; Johnson 2014). Sin embargo, mientras que nuestro conocimiento sobre los mecanismos y patrones de participación política a nivel individual es considerable, se ha prestado mucho menos atención a otros tipos de participación indirectamente vinculados con la política (Lehman et al. 2012).

Diversos estudios han alertado sobre la caída o la falta de participación y el desinterés y la desconfianza de los ciudadanos en la política institucional, tanto en democracias consolidadas, como en democracias más jóvenes (Putnam 2000; Wattenberg 2002; Macedo et al. 2005). De hecho, la caída en la participación y el incremento de la apatía no son fenómenos nuevos. A fines del siglo pasado, Skocpol y Fiorina (1999), por ejemplo, ya advertían una tendencia hacia una menor participación en la vida pública y una mayor indiferencia hacia el sistema democrático. En el caso de América Latina, Latinobarómetro ha encontrado sistemáticamente un patrón de baja participación y una tendencia creciente hacia la indiferencia. Por ejemplo, en 1995, solo 16\% de los latinoamericanos se decía indiferente entre la democracia y un sistema autoritario. Poco más de dos décadas después, en 2018, la proporción de latinoamericanos indiferentes aumentó a $28 \%$.

Las explicaciones de estos patrones a menudo convergen en la discusión sobre el desencanto con la democracia y cómo éste tiende a traducirse en apatía y falta de participación (O'Toole et al. 2003; Stoker 2006; Hay 2007). Sin embargo, los estudios rara vez hablan sobre tipos de participación no estrictamente política o partidista, como por ejemplo el trabajo voluntario para resolver un problema de la comunidad o para mejorar las condiciones de vida de un grupo social específico. Se conoce poco sobre la falta de participación en sí misma y cómo se relaciona no sólo con la apatía, sino también con la alienación y las oportunidades limitadas, la desigualdad, la desconexión con la política partidaria y la desconfianza en las instituciones (Lehman et al. 2012). De igual manera, se sabe poco sobre la relación entre la falta de participación política y su substitución por otros tipos de participación ciudadana, como es el caso de la participación comunitaria, que se refiere a actividades que intentan incidir sobre la sociedad (organizar eventos o resolver problemas comunitarios), y que no están directamente relacionados con el poder o los partidos políticos. 
La intención del presente estudio es analizar en conjunto diversas formas de participación, además de la meramente política. Nos concentramos en México pues nos parece un caso interesante porque se trata de la democracia hispanohablante más grande del mundo y porque, al igual que otras naciones latinoamericanas, enfrenta retos importantes: pobreza y alta desigualdad, corrupción rampante, crimen organizado, violaciones de derechos humanos y baja productividad y competitividad que han dado lugar al surgimiento de liderazgos populistas. Paradójicamente, durante las últimas décadas, México ha mantenido tasas de participación electoral nada despreciables que oscilan entre $60 \%$ y $65 \%$, combinadas con altos niveles de desconfianza en los políticos y partidos tradicionales (INE, 2014). Más aún, a pesar de las altas tasas de participación electoral, otros tipos de participación política son bajos y cada vez menos frecuentes (Klesner 2009; INE 2014). Y, sin embargo, aunque los mexicanos participan poco políticamente más allá de lo electoral, sí se involucran de manera importante en actividades comunitarias. Es decir, el caso mexicano nos permite distinguir, de manera muy clara, las diferencias que existen entre los distintos tipos de participación ciudadana electoral, política y comunitaria.

Nuestro propósito es construir una tipología de los tipos de participación que reportan los ciudadanos, bajo el supuesto de que estos tipos pueden tener un efecto diferenciado en la calidad democrática de la sociedad. Empíricamente, usamos datos del Informe País sobre la Calidad de la Ciudadanía en México que hasta ahora no han sido analizados tipológicamente. El presente estudio contribuye al debate sobre participación y apatía ciudadanas con una tipología empíricamente validada que arroja nuevas preguntas a la luz de patrones diferenciados de participación política y comunitaria.

El artículo está organizado de la siguiente manera: en la siguiente sección, presentamos datos sobre la participación política en México en perspectiva comparada. En la segunda sección, discutimos cuestiones teóricas. La tercera sección describe el diseño, metodología y datos usados en nuestra investigación. La cuarta sección presenta la tipología y sus bases. La quinta sección concluye.

\section{PARTICIPACIÓN EN MÉXICO}

El voto es la forma más elemental de participación política en una democracia. Es la forma más sencilla y la que menor costo implica para el ciudadano común. Sin embargo, en México, al igual que en otros países latinoamericanos, de principios de los noventa hasta hoy, el abstencionismo ha tenido una tendencia al alza. Con todo, los niveles de votación de México no distan de los de varias democracias consolidadas. Comparativamente, México (65.62\% de voto en el $2018)^{1}$ tiene porcentajes de votación parecidos a los de Alemania $(69.11 \%, 2017)$, España $(65.14 \%, 2019)$, Reino Unido $(62.88 \%, 2017)$ y está por arriba de las cifras

La cifra se refiere al porcentaje de participación electoral en la elección legislativa de diputados del Congreso nacional. 
de Chile $(49.55 \%$, 2017), Estados Unidos $(55.98 \%, 2016)$ o Francia $(38.62 \%, 2017)$. Algunos casos muy distantes del caso mexicano son el de Argentina (77.69\%, 2017) o Uruguay $(98.29 \%, 2014)$, en donde el voto es obligatorio (con sanción). La tendencia abstencionista en América Latina y otras regiones del mundo ha estado acompañada por un descrédito del sistema político y, en especial, de los partidos políticos. Estos han sido incapaces de generar representatividad y confianza en los ciudadanos, sobre todo entre los jóvenes: por ejemplo, $52 \%$ de los mexicanos de 18 a 29 años no se identifica con ningún partido (INE 2014).

Sin embargo, el voto es solo una de las diferentes modalidades de participación política que un individuo puede ejercer. Existen otros tipos de participación que van desde firma de peticiones, contacto con autoridades gubernamentales, el involucramiento en campañas electorales, mítines, boicots o caravanas, hasta actos disruptivos como la ocupación de edificios públicos, el bloqueo de lugares públicos, e incluso actos encaminados a derrocar al gobierno.

Desde 2000, las investigaciones que han intentado explicar el voto en México han ido creciendo de manera sustantiva. No obstante, no ha sucedido lo mismo con la participación no electoral. Existe un grupo importante de trabajos sobre movimientos sociales y de protesta, sobre todo desde perspectivas sociológicas y antropológicas (Davis 1991; Escobar y Álvarez 1992; Bensusán y Middlebrook 2013; Béjar 2015; Ramírez 2016; Inclán 2018), pero hay pocos estudios que intenten explicar y tipificar la participación no electoral con datos de encuestas (Klesner 2009).

En el caso mexicano, aunque aún hay pocos datos para comparar la evolución de las distintas formas de participación no electoral, pareciera que los tipos de participación directa han ido a la baja. Como puede verse en la tabla 1, distintos tipos de acción política han tenido una tendencia negativa en un periodo de 10 años. Las bajas más drásticas se dieron en boicots, manifestaciones y firma de peticiones. Es interesante observar que las cifras más altas se presentan antes del 2000, año en que el Partido Revolucionario Institucional (PRI) perdió la Presidencia de la República por primera en la historia del país.

Tabla 1. Participación política no electoral en México

\begin{tabular}{lrrrrr}
\hline & $1990-94$ & $1995-99$ & $2000-2004$ & $2005-09$ & 2010-14 \\
\hline Firma de petición & 31.4 & 28.3 & 14.8 & 19.7 & 18.3 \\
Boicot & 6.0 & 8.6 & 1.7 & 2.7 & 2.5 \\
Manifestación & 20.2 & 9.6 & 3.4 & 15.7 & 10.2 \\
Huelga & 6.6 & 5.6 & 2.3 & 2.3 & 5.5 \\
Ocupación de edificio público & 4.6 & 4.2 & 1.8 & - & 4.2 \\
\hline
\end{tabular}

Se muestra porcentajes de la población que ha participado en alguna modalidad de participación no electoral. Fuente: World Values Survey, 1990/94-2010/14. 
Como hemos dicho, la tasa de participación electoral mexicana no es muy distinta a la de otras democracias latinoamericanas, o incluso de democracias consolidadas. En cambio, en relación a otros tipos de participación política no electoral como la firma de peticiones, boicots, huelgas o manifestaciones, los mexicanos parecen involucrarse mucho menos que los ciudadanos de países como Estados Unidos, Alemania, Suecia, Brasil o Chile (Figura 1). Más aún, Martí y Llamazares (2011) encuentran que, en México, la escasa participación política de protesta está notablemente asociada a la política partidaria, hasta el punto que puede afirmarse que la política disruptiva la impulsan los propios partidos cuando les es conveniente.

Figura 1. Participación política no electoral en distintos países (porcentajes)

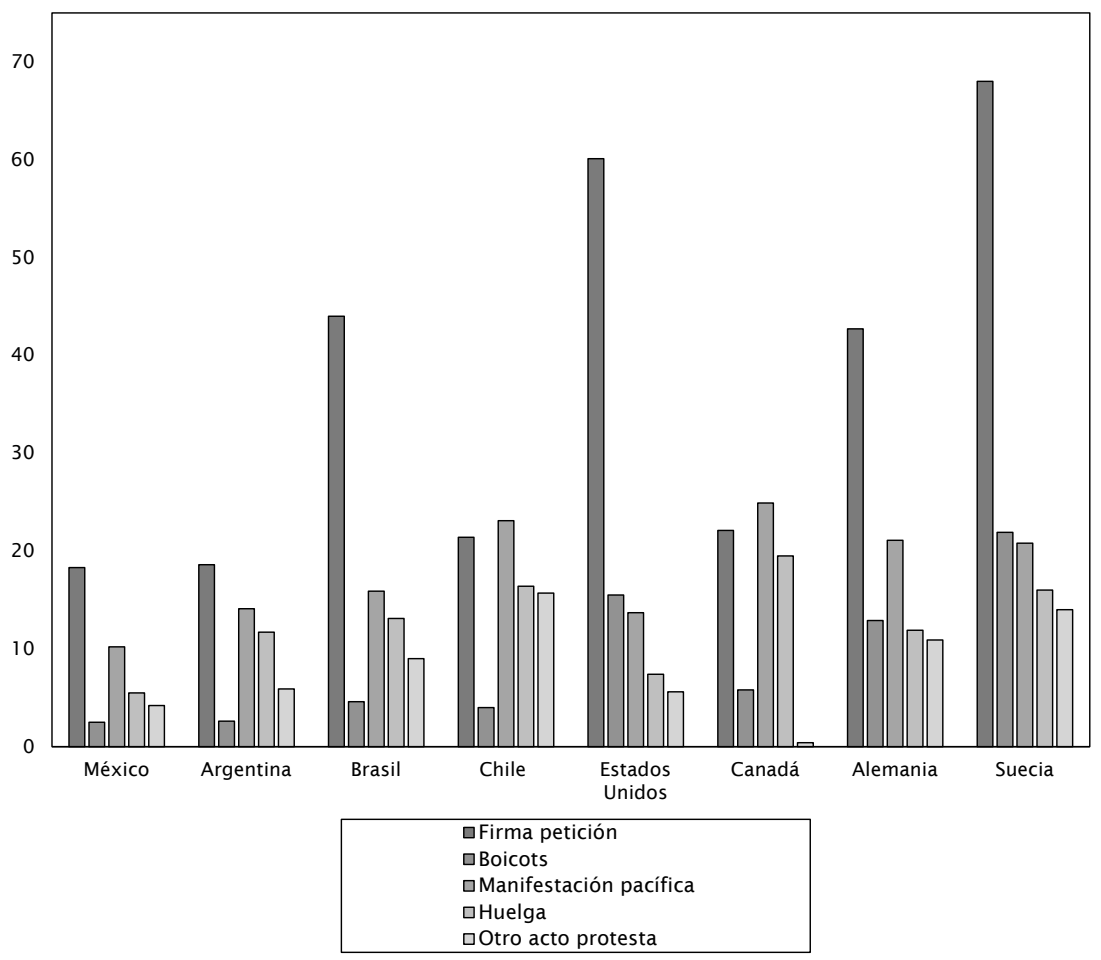

Fuente: World Values Survey 2010-2014

No obstante, es muy importante señalar que existen otros tipos de participación que no se vinculan directamente con la política o los partidos y que, sin embargo, sí representan formas de involucramiento cívico. Estas actividades, aunque no tienen el objetivo inmediato de incidir en la política o en los actores políticos, bien pueden ser relevantes para la acción colectiva y la calidad de la convivencia social. Este tipo de formas de participación incluyen actividades de tipo comunitario como ayudar a resolver un problema o participar en trabajo voluntario. Si se comparan las figuras 1 y 2, es claro que la participación comunitaria tiende a ser más frecuente entre los mexicanos que la política. 
Figura 2. Participación comunitaria en distintos países (porcentajes).

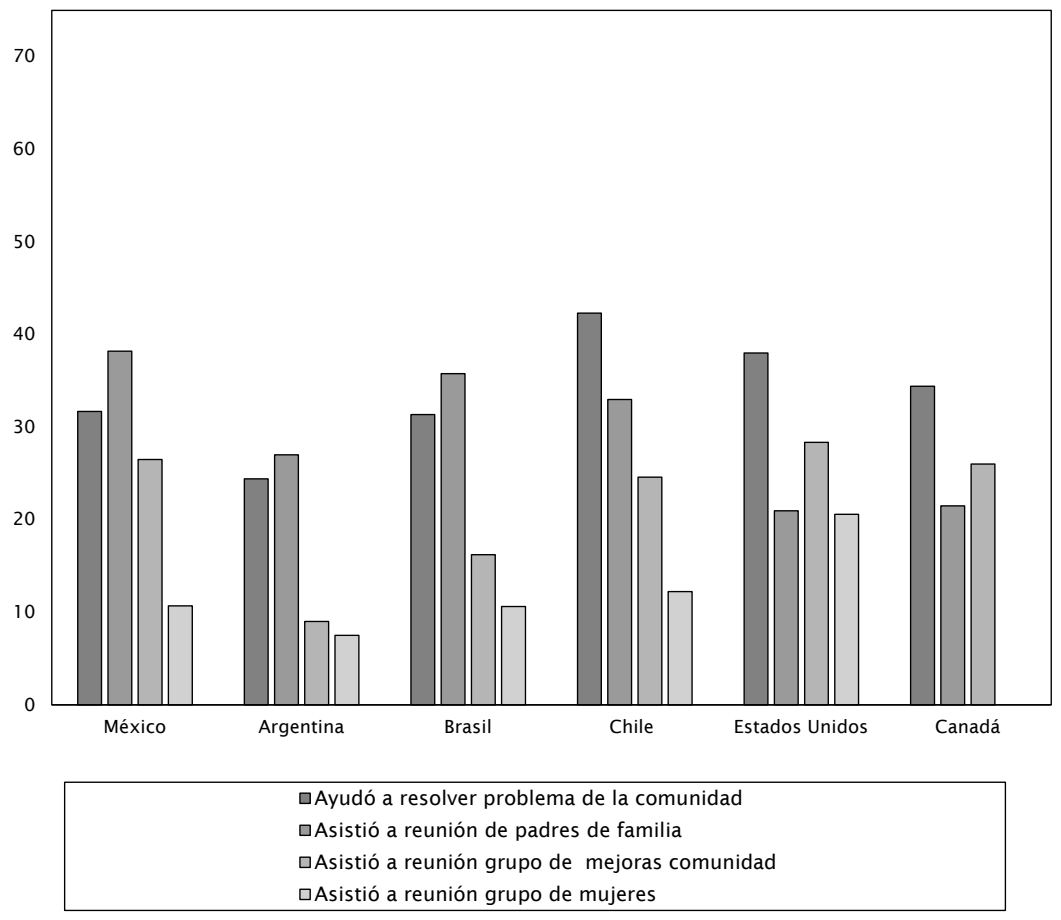

Fuente: LAPOP 2014

Como se muestra en la figura 2, las tasas de participación de los mexicanos en actividades no estrictamente políticas como son la ayuda a la comunidad, la asistencia a una reunión de padres de familia o un grupo de mujeres, son muy parecidas a las de democracias como Canadá, Chile o Estados Unidos y, en algunos rubros, incluso mayores. Es decir, si nos centramos en la participación estrictamente política, podemos decir que las tasas de participación son preocupantemente bajas. Sin embargo, si se consideran otros tipos de participación ciudadana, las conclusiones pueden ser distintas.

\section{CUESTIONES TEÓRICAS}

La teoría democrática ha destacado históricamente la participación política, especialmente la electoral. Por medio de la participación política, los ciudadanos en una democracia buscan controlar quién ocupa cargos públicos y, además, intentan ejercer influencia sobre las políticas públicas (Milbrath y Goel 1977; Teorell 2006). Se espera que los ciudadanos participen activamente porque mediante la deliberación pública y la acción colectiva e individual se definen los objetivos de la sociedad, así como los cursos de acción pública que resultan más aceptables (Almond y Verba 1963; Verba et al. 1995; Dalton 2008). 
Los estudiosos del fenómeno han ofrecido definiciones alternativas de participación política. Por ejemplo, Booth y Seligson (1978) definen participación política como el comportamiento que influye o que intenta influir en la distribución de bienes públicos. En un tono similar, Weiner (1971: 164) define participación política como "cualquier acción voluntaria, exitosa o no, organizada o no, intermitente o continua, que utiliza medios legítimos o ilegítimos para influir en la elección de políticas públicas, la administración de los asuntos públicos, o la selección de líderes políticos a cualquier nivel de gobierno, local o nacional". Finalmente, Brady (1999: 737) argumenta que la participación política es "todas aquellas actividades cuya intención es influir en los resultados políticos al ir dirigidas a llamar la atención de las élites políticas y sociales relevantes".

Independientemente de la definición adoptada, la participación política sigue teniendo un papel preponderante en las teorías sobre ciudadanía. Sin embargo, existen otras formas de participación no estrictamente política que pueden desempeñar un papel importante para la gobernabilidad democrática (De Weerd et al. 2005; Van Nierop 2006). Existen acciones que pueden verse como formas "latentes" o marginales de comportamiento político (Ekman y Amnå 2012). Estas acciones se refieren a actos cívicos como el trabajo voluntario, la cooperación comunitaria o las acciones altruistas, como la donación en dinero o especie. Este tipo de participación, que nosotros llamamos comunitaria, puede definirse como "aquellas actividades del ciudadano común que intentan ejercer influencia sobre la sociedad y que es relevante para otros, más allá de su propia familia o amigos cercanos" (Adler y Gogin 2005: 241). Nuestro argumento es que, para poder tener un análisis completo sobre la participación ciudadana en una sociedad, es importante considerar formas de participación comunitaria y no solamente la estrictamente política. Más aún, es importante entender cómo se vinculan formas de participación comunitaria con la participación política y cuáles son los tipos de participación que pueden surgir de las distintas combinaciones posibles.

\section{Participar o no participar}

Las explicaciones de la participación tradicionalmente se derivan de una serie de factores personales y factores del entorno sociopolítico de los ciudadanos. El modelo clásico de Verba y sus colegas (1995), por ejemplo, se basa en tres conjuntos de factores para explicar la participación: motivos, capacidades y oportunidades de movilización. Otros estudios han propuesto la idea de que la participación no solo depende de esos factores, sino también de cómo las políticas públicas influyen en las actitudes de los ciudadanos hacia el desempeño del gobierno y hacia ciertos grupos sociales (Campbell 2006).

Otro argumento relativamente común en la literatura sobre participación es que las personas tienden a participar en política no solo en función de sus motivos, recursos o las oportunidades de movilización, sino que no deben ser in- 
diferentes hacia el sistema político y, de manera más general, hacia la esfera de lo público (Hay 2007). La indiferencia, en este sentido, conlleva sentimientos de alienación y desafección, así como lejanía y abandono del sistema político y sus instituciones. Esto implica que la indiferencia puede ser permanente y, por tanto, puede imposibilitar la participación (Di Palma 1970; Dalton 2008). Sin embargo, la falta de participación también puede desencadenarse por la insatisfacción con la política y el desempeño institucional. La insatisfacción se relaciona con una aversión ante una situación o decisión. Distinta de la indiferencia, la insatisfacción puede ser manejable, temporal y motivacional. Más aún, la insatisfacción puede estimular la participación si la gente cree que puede afectar el estatus quo (Di Palma 1970; Castillo et al. 2015).

La distinción entre insatisfacción e indiferencia es importante. Estos representan dos factores distintos que pueden relacionarse de maneras concretas con la participación o la falta de ésta. Se puede suponer que las personas tenderán a no involucrase si son indiferentes hacia lo público. Los ciudadanos indiferentes tienen menos probabilidades de participar porque están alienados y es posible que ni siquiera se sientan identificados con alguna forma de comunidad cívica (Di Palma 1970). Pero las personas insatisfechas con una situación podrían participar si, en circunstancias dadas, ven una oportunidad de cambio por medio de su involucramiento.

Los puntos anteriores ofrecen una visión general de los factores y mecanismos explicativos de la participación. Para analizar simultáneamente los patrones de participación de los mexicanos, suponemos, en primer lugar, que la participación puede ocurrir dentro o fuera de la arena política. Es decir, la falta de participación política, como se definió arriba, no implica la falta de participación ciudadana. Esto se deriva de la observación de que ciudadanos indiferentes o insatisfechos pueden recurrir a otras formas de activación cívica que no necesariamente están relacionadas con las instituciones políticas formales, como lo son distintas formas de participación comunitaria (Ekman y Amnå 2012; Teorell 2006). Partiendo de ese supuesto y si cruzamos las dos dimensiones (participación política y participación comunitaria), podemos identificar al menos cuatro tipos de participación ciudadana (Figura 3).

Empezando por el cuadrante superior derecho de la Figura 1, el tipo II representa un perfil caracterizado por participación en actividades políticas como votar, participar en partidos políticos, firmar peticiones o asistir a reuniones del cabildo municipal, participar en manifestaciones públicas, bloqueos o huelgas, y también participación en actividades comunitarias, como donar dinero a la Cruz Roja, dar alimentos, medicinas a personas pobres o hacer trabajo voluntario. Por el contrario, el tipo III representa personas que no participan en ningún tipo de actividad, ya sea política o comunitaria. Siguiendo nuestro argumento, el perfil de participación tipo III sería propiamente apático, mientras que el tipo II representa a ciudadanos "hiperactivos" como los que se encuentran en algunas versiones de la teoría democrática tocquevilliana o de la democracia participativa (Pateman 1970; Rousseau 1973; Mill 1977). No obstante, lo intere- 
Figura 3. Tipos de participación ciudadana

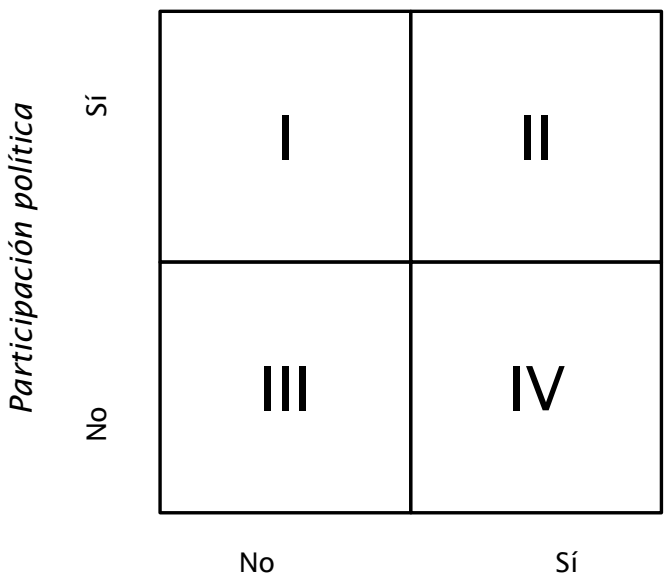

Participación comunitaria

sante es que lógicamente existen al menos dos tipos adicionales de participación. El tipo I se refiere a un perfil de participación orientado a asuntos políticos y que evita otros tipos de activación como las actividades comunitarias. Éste sería el tipo convencional que se teoriza en la mayoría de los estudios clásicos sobre ciudadanía. En cambio, el tipo IV representa a los ciudadanos que participan predominantemente en actividades comunitarias como las descritas arriba, pero que tienden a evitar la participación política. En este último caso, uno podría esperar una mayor frecuencia de ciudadanos alienados del sistema político que deciden volcarse por formas de participación fuera de la política institucional o de protesta. Vale la pena subrayar el punto: los ciudadanos caracterizados por un perfil de participación con el del cuadrante IV siguen siendo ciudadanos participativos, aunque apolíticos, y son distintos de los propiamente apáticos.

En principio, la ciudadanía democrática se identifica con la participación activa de los ciudadanos. Esto puede ir desde la mera participación en procedimientos y actividades políticas convencionales (ciudadanos tipo I) hasta ciudadanos que participan en múltiples actividades políticas y comunitarias (ciudadanos tipo II). Por lo tanto, la calidad de los regímenes democráticos a menudo se concibe, al menos en parte, condicionada a la participación de los ciudadanos. La tesis central es que se necesitan ciudadanos activos dentro y fuera del sistema político para monitorear el proceso político y para catalizar o resistir el cambio social, político e institucional (Norris 1999). La teoría democrática, particularmente en sus versiones más clásicas, destaca la participación activa como condición para que una democracia funcione bien. En otras palabras, utilizando la tipología anterior, una alta frecuencia de ciudadanos con un perfil de participación tipo III debería ser motivo de preocupación, al menos desde el mirador de la calidad de las instituciones democráticas. 
Los tipos anteriores son útiles, como veremos más adelante, para distinguir patrones agregados de participación y apatía. Además, uno podría esperar diferentes determinantes para cada tipo; es decir, diferentes patrones de participación pueden estar asociados con distintas características y patrones en términos de motivos, capacidades y factores de movilización. Las variaciones con respecto a la falta de participación (política y comunitaria) también podrían responder a determinantes específicas. En este sentido, los distintos tipos de participación ciudadana pueden reflejar diferentes estructuras de variables sociales y políticas. Pero antes de explorar estos puntos, es relevante primeramente verificar si los tipos identificados arriba se corresponden con patrones observables en datos empíricos.

\section{TIPOS Y MEDIDAS DE PARTICIPACIÓN}

La validación empírica de la tipología se enfoca en diferentes formas de participación política y comunitaria de ciudadanos mexicanos (18 años o más) en 2013. La muestra consistió en $\mathrm{N}=8157$ ciudadanos, recopilada como parte del Informe País sobre la Calidad de la Ciudadanía en México. Los datos fueron recolectados mediante encuesta en hogares, utilizando un cuestionario estandarizado (INE 2014). Alrededor de 55\% de la muestra son mujeres. La edad promedio es 42 años (SD = 16.2), y el ingreso medio (por hogar) equivale a un rango de 1-126 USD por mes. Los encuestados en la muestra tienen un nivel educativo medio equivalente a la escuela secundaria incompleta (aproximadamente 8 años de escolaridad).

\section{Participación}

Para capturar patrones de participación política, usamos medidas retrospectivas de participación política convencional y de protesta (cf. Kam 2012). Se preguntó a los encuestados si habían participado en diferentes actividades en los últimos 12 meses. Las formas convencionales de participación que se midieron fueron (1) hablar sobre política con otros, (2) convencer a otros de votar por un candidato, (3) participar en las actividades de un partido político, y (4) asistir a reuniones del cabildo municipal. La respuesta para cada actividad fue codificada dicotómicamente: 1: Sí ha participado, 0: No ha participado. Excluimos el voto de nuestra medida de participación política convencional. Aunque votar es la forma más prominente (y a veces la única) de participación política en países democráticos (O'Toole et al. 2003), ésta también implica instituciones y restricciones específicas que son poco comunes o inexistentes para otras formas de participación (por ejemplo, un calendario electoral y registro de votantes en un padrón electoral, cf. Nickerson 2014). Por lo tanto, en este estudio, tratamos la participación electoral de manera separada y como co-variable de otras formas de participación política. 
Para medir otras formas de participación política (protesta), usamos las siguientes actividades: (1) firmar peticiones a políticos o funcionarios públicos, (2) participar en manifestaciones públicas, (3) participar en bloqueos, boicots $u$ ocupar espacios públicos, y (4) participar en una huelga. En todos los casos, la respuesta fue codificada dicotómicamente: 1: Sí han participado, 0: No han participado. Las formas de participación política de protesta se distinguen de las "convencionales" en que son menos comunes y tienden a actuar en los márgenes o a transgredir el estatus quo institucional. La protesta es usualmente disruptiva y más costosa (individual y colectivamente) que la participación política convencional (Fiorina 1999; Dalton 2008).

Para medir la participación comunitaria, usamos las siguientes actividades: (1) donar a la Cruz Roja, (2) haber donado comida, medicinas o ropa, (3) haber hecho trabajo comunitario voluntario (no remunerado) y (4) ayudar a un miembro de la comunidad sin esperar gratificación. Para cada actividad, la respuesta fue codificada dicotómicamente (1: Sí lo han hecho, 0: No lo han hecho). En línea con las consideraciones teóricas arriba, estas medidas capturan formas de involucramiento que intentan ejercer influencia sobre la sociedad, sin ser formas de participación estrictamente política (sea convencional o de protesta política).

En total, se utilizaron doce medidas de participación: cuatro de participación política convencional, cuatro de participación política de protesta y cuatro de participación comunitaria. Los ítems referidos a diferentes tipos de participación fueron sujetos a un análisis de componentes principales con rotación oblicua (Tabla 2). Las pruebas de adecuación de la muestra resultaron satisfactorias, $^{2}$ y se extrajeron tres componentes usando el criterio Káiser. ${ }^{3}$

Tabla 2. Matriz de estructura de componentes principales tras rotación oblicua

\begin{tabular}{|c|c|c|c|}
\hline & \multicolumn{3}{|c|}{ Componentes a } \\
\hline & 1 & 2 & 3 \\
\hline Hablar sobre política & & & 0.68 \\
\hline Convencer a otros de votar & & & 0.70 \\
\hline Participar en un partido político & & & 0.69 \\
\hline Asistir a las reuniones del cabildo & & & 0.52 \\
\hline Firmar peticiones a servidores públicos & 0.56 & & 0.33 \\
\hline Participar en manifestaciones & 0.72 & & \\
\hline Participar en bloqueos, boicots u ocupaciones & 0.72 & & \\
\hline Participar en una huelga & 0.67 & & \\
\hline
\end{tabular}




\begin{tabular}{|c|c|c|c|}
\hline & \multicolumn{3}{|c|}{ Componentes $^{\text {a }}$} \\
\hline & 1 & 2 & 3 \\
\hline Donar a la Cruz Roja & & 0.64 & \\
\hline Donar alimentos, medicinas o ropa & & 0.75 & \\
\hline Trabajo comunitario (sin paga) & & 0.56 & \\
\hline Ayudar a alguien de la comunidad & & 0.70 & \\
\hline Valor propio $(\lambda)$ & 1.88 & 1.82 & 1.80 \\
\hline Proporción de varianza (\%) & 16 & 15 & 15 \\
\hline
\end{tabular}

a Solo se reportan correlaciones $\geq 0.3$

$N=8,157$

El primer componente se caracteriza por fuertes correlaciones con formas de participación política de protesta. El segundo componente está altamente correlacionado con formas de participación comunitaria. Finalmente, el tercer componente se caracteriza por una fuerte correlación con formas convencionales de participación política. Las correlaciones parciales entre los componentes fueron todas positivas y estadísticamente significativas $(p<0.05)$. La participación comunitaria está relacionada moderadamente con la protesta política $(r=0.16)$ y solo marginalmente con la participación política convencional ( $r=0.07)$. Como es de esperarse, la participación política de protesta y la participación política convencional están correlacionadas $(r=0.25)$. Con base en los componentes extraídos, calculamos puntajes de participación usando mínimos cuadrados ordinarios (OLS).

\section{ANÁlISIS TIPOLÓGICO}

Con base en los puntajes individuales de participación, se creó una tipología empírica para estudiar los diferentes perfiles de participación en la muestra. Realizamos un análisis de conglomerados de $\mathrm{k}$ medias con un procedimiento iterativo de clasificación y 25 puntos de inicio aleatorios (Aldenderfer y Blashfield 1984; Lander 2014). El análisis de conglomerados permite clasificar a los individuos en diferentes tipos, en función de sus puntajes de participación política convencional, participación política de protesta y participación comunitaria. Los encuestados se clasificaron en tipos (conglomerados) que minimizaban las diferencias entre individuos, mientras que se maximizaban las diferencias entre grupos. Se retuvo una solución de cuatro grupos $(k=4) .{ }^{4}$

El puntaje medio en cada medida de participación para cada conglomerado identificado se presenta en la tabla 3. Al primer grupo se denominó con- 
vencional, ya que estos individuos predominantemente participan en formas convencionales de participación política, seguido por formas comunitarias de participación, mientras que evitan la protesta política (cf. tipo I, arriba). El segundo grupo, ciudadanos apáticos, tiene puntuaciones medias negativas en todos los factores, lo que indica aversión a todas las formas de participación política y comunitaria (cf. tipo III). El tercer grupo, ciudadanos apolíticos, está formado por individuos que típicamente participan en actividades comunitarias, pero evitan formas de participación política convencional y de protesta (cf. tipo IV). Finalmente, el cuarto grupo se llamó hiperactivos (cf. tipo II), ya que estos individuos participan en todas las formas de participación, pero participan con mucha mayor frecuencia que otros en protestas políticas. De la muestra, $16 \%$ fue clasificado como convencional, ${ }^{5} 35 \%$ apático, ${ }^{6} 45 \%$ apolítico, ${ }^{7}$ y $4 \%$ hiperactivo. ${ }^{8}$

Tabla 3. Patrones de participación por tipo de ciudadanos

\begin{tabular}{lrrrr}
\hline & \multicolumn{4}{c}{ Tipos de ciudadanos } \\
\cline { 2 - 5 } & Convencional & \multicolumn{1}{c}{ Apático } & Apolítico & Hiperactivo \\
\hline Participación convencional & 1.73 & -0.44 & -0.38 & 1.09 \\
Protesta política & -0.09 & -0.19 & -0.19 & 3.94 \\
Participación cívica & 0.26 & -1.05 & 0.69 & 0.30 \\
N & 1,298 & 2,845 & 3,661 & 353 \\
\hline
\end{tabular}

La evidencia del análisis de conglomerados apoya la tipología teorizada; sin embargo, ofrece algunos matices que serán discutidos más abajo. Además, realizamos análisis complementarios para validar la robustez de la solución de conglomerados. Para cada réplica (10 en total), usamos una submuestra aleatoria de $\mathrm{N}=5,000$ observaciones. En todos los casos, encontramos estructuras de conglomerados idénticas o muy similares. Además, las soluciones de cuatro conglomerados en las diez submuestras coincidían estrechamente con la clasificación del análisis agrupado (promedio ponderado de $x=0.98$ ), y todos los valores kappa individuales (0.96-0.99) exceden los niveles aceptables de replicación, lo que indica que los resultados obtenidos son estadísticamente robustos (Aldenderfer y Blashfield 1984). 


\section{Diferencias entre tipos}

Para explorar las diferencias entre perfiles de participación, se realizó un análisis de regresión multinomial. El propósito fue analizar las características individuales relacionadas con diferentes tipos de participación. Las variables de contraste corresponden, así, a diferencias en motivos, capacidades y oportunidades de movilización, así como a medidas de indiferencia e insatisfacción con la democracia y la política.

Para participar, los individuos deben tener primero algún tipo de motivación, especialmente si se admite que la participación es costosa (Leighley 1995; Kam 2012). Desde un punto de vista analítico, los motivos son una condición previa para la participación en la medida en que los individuos se orientan a múltiples objetivos y porque la orientación de estos hacia la acción colectiva es temporal y espacialmente débil (Lindenberg 2013). No obstante, las motivaciones orientadas hacia lo público pueden variar en un continuo que va desde motivos utilitarios (por ejemplo, las personas quieren proteger sus recursos) a motivaciones normativas (por ejemplo, las personas desean cumplir con sus deberes cívicos; véase Campbell 2006). Ejemplos de motivos en un lado son la existencia de confianza en el sistema o la creencia de que los recursos de uno están asegurados (cf. Bateson 2012). Del otro lado, el compromiso ideológico o altos costos morales derivados de romper las reglas del juego democrático, son ejemplos de razones que surgen de un sentido del deber cívico internalizado (Fennema y Tillie 1999).

Además de la motivación, las diferentes capacidades de los individuos potencian o restringen las posibilidades de participación, dependiendo del tipo de acciones en las que haya que involucrarse. Cada tipo de participación difiere en los requisitos que plantea al participante; es decir, distintas formas de participación refieren a distintas estructuras de costos (Brooks 2014). En este sentido, Verba y sus colegas (1978) clasificaron las diferencias entre las formas de participación según cinco criterios: (1) si el acto transmite información sobre las preferencias políticas del individuo o si ejerce presión para su cumplimiento, (2) si el acto se orienta hacia un resultado social amplio o un interés particular, (3) el grado potencial de conflicto involucrado en la actividad, (4) la cantidad de esfuerzo requerido, y (5) la cantidad de cooperación colectiva requerida para actuar. Estos criterios implican que las diferencias en la dotación de recursos tangibles e intangibles de los individuos se pueden traducir en distintos patrones de participación. Es decir, ciudadanos con diferentes recursos probablemente participarán de maneras diferentes (Brooks 2014). Por tanto, la disponibilidad de recursos como la educación, el ingreso o el tiempo libre pueden explicar diferencias en la frecuencia y tipo de participación (Leighley 1995).

Los grupos, las redes sociales, la organización social y, de manera más general, las oportunidades de movilización también son importantes para comprender la participación (McClurg 2003). Las personas que tienen motivación y capacidad para volverse activas tienen más probabilidades de hacerlo si éstas son 
movilizadas o incorporadas a organizaciones (Rosenstone y Hansen 1993). La movilización se cruza con la participación de distintas maneras. Fundamentalmente, sin embargo, la movilización mediante actividades de organizaciones constituye una fuente crucial de socialización y acción colectiva en todas las democracias (Verba et al. 1978). Los indicadores de identificación con colectivos $\mathrm{u}$ organizaciones pueden indicar una propensión hacia la participación. Además, la membresía en grupos sociales, cívicos o de voluntarios también puede estimular la participación comunitaria. Varios autores han argumentado que la experiencia en la toma de decisiones en este tipo de organizaciones desarrolla habilidades y actitudes que pueden trasladarse a la arena pública (Brady et al. 1995; Putnam 1995). Los grupos y organizaciones también pueden movilizar activamente a sus miembros o al menos proporcionar puntos de entrada para conocer formas de participación (Verba et al. 1978; Verba et al. 1995; Ayala 2000). Asimismo, la literatura sobre movimientos sociales afirma que los individuos pueden tener diversos incentivos materiales o ideológicos para involucrarse, pero la decisión última de participar se origina como respuesta al cambio en las oportunidades y limitaciones a la acción colectiva (Tarrow 1998; Tilly 1978). En otras palabras, los ciudadanos son más propensos a participar si se les anima a hacerlo: una situación que provee oportunidades para la movilización favorece la existencia de ciudadanos activos (Quintelier y Van Deth 2014).

\section{Co-variables de participación}

Motivaciones. Dadas las consideraciones anteriores, quisimos primeramente capturar la percepción sobre el Estado de Derecho como proxy de confianza en la imparcialidad del sistema político. Esta percepción se midió con la pregunta “¿En qué medida cree que se respetan las leyes en México?” Las categorías de respuesta variaron de 0: No se respetan nada, a 3: Se respetan mucho. La confianza institucional se midió preguntando hasta qué punto se confiaba en una serie de siete instituciones públicas (jueces, policía, gobierno federal, ejército, congreso, gobierno estatal y gobierno municipal). La respuesta para cada ítem varió de 0: No confía nada, a 3: Tiene mucha confianza. La variable que finalmente se incluyó en el análisis es la sumatoria de los siete ítems. Otra motivación potencialmente relevante se refiere a la seguridad pública. Ésta puede incidir tanto en la confianza en el sistema como en la creencia de que los recursos propios están asegurados. Para medir lo anterior, usamos una pregunta de victimización: “¿En los últimos 12 meses, usted o algún miembro de su hogar ha sido víctima de un delito?" La respuesta se codificó dicotómicamente, 1 en caso afirmativo ( 0 , de lo contrario).

Capacidades. Un primer elemento a considerar es la eficacia política. Ésta se refiere a la percepción del ciudadano de la probabilidad de que sus acciones le traerán mayores beneficios que los costos en los que incurra y que su participación en los asuntos públicos puede tener un impacto real en el resultado. Esta variable se midió con la pregunta “¿En qué medida está de acuerdo con 
la afirmación 'Las personas como yo tienen influencia sobre el gobierno'?" La respuesta se codificó en una escala de 5 puntos que va de 0: Totalmente en desacuerdo a 4: Totalmente de acuerdo. Para contrastar distintas capacidades, entendidas como perfiles socioeconómicos (cf. Verba et al. 1995), incluimos las siguientes características: ingreso del hogar, sexo, nivel educativo, estado de desempleo y edad.

Oportunidades de movilización. Medimos la identificación con un partido político con la pregunta "Independientemente de por cuál partido votó, ¿normalmente se identifica con [nombre del partido político]?" Utilizamos los tres principales partidos políticos nacionales en 2013 (Partido Revolucionario Institucional, Partido Acción Nacional y Partido de la Revolución Democrática). Tres variables dicotómicas, una por partido político, indican identificación partidaria. La membresía en organizaciones ciudadanas —una indicación de acceso a capital político y social- se midió preguntando a los encuestados si eran miembros de un grupo estudiantil, profesional, político, religioso, deportivo, cultural, de padres o si pertenecían a alguna organización de caridad, acción humanitaria, de derechos humanos, ambientalista o de vecinos. Si los encuestados respondieron sí a uno o más de los ejemplos mencionados, su respuesta se codificó 1 ( 0 , de lo contrario).

Otras variables. Voto es una variable dicotómica que indica si la persona votó o no en las elecciones federales de julio de 2012. La insatisfacción con la democracia se midió con la pregunta: “¿Está de acuerdo con la afirmación ‘En una democracia, muchos individuos participan, pero solo unos pocos se benefician'?" (Castillo et al. 2015). La indiferencia se midió con la pregunta: “¿Está de acuerdo con la afirmación 'A la gente como yo no le importa si México es una democracia o no'?" La respuesta a ambas preguntas se codificó dicóticamente (1: Estoy de acuerdo, 0: No estoy de acuerdo).

Usamos la tipología empírica como variable dependiente. Modelamos la probabilidad de pertenecer a uno de los perfiles de la tipología (convencional, apolítico, apático o hiperactivo) con base en las co-variables. Es decir, el análisis multinomial (tabla 4) proporciona información sobre las variables relacionadas con la probabilidad de pertenecer a un tipo determinado. Para hacer este contraste, usamos la categoría de participación convencional como grupo de referencia. Mediante razones de verosimilitud podemos estimar la significancia estadística de los efectos de todos los predictores incluidos en el modelo, en todas las categorías de respuesta (ver Bilder y Loughin 2015). Este análisis (deviance analysis) muestra que los datos observados serían extremadamente improbables si los efectos de las co-variables fueran, de hecho, cero. Esto es, existe evidencia estadística a priori de que este conjunto de variables independientes (motivos, capacidades, oportunidades de movilización y variables adicionales) puede ayudarnos a explicar diferencias entre los cuatro perfiles de la tipología. 
Tabla 4. Análisis multinomial para distintos tipos de participación

\begin{tabular}{|c|c|c|c|c|}
\hline Tipo $^{\mathrm{a}}$ & & $\beta$ & Error & $\operatorname{Exp}(\beta)$ \\
\hline \multirow[t]{20}{*}{ Apolítico } & Intercepto & $2.78^{* * *}$ & 0.21 & - \\
\hline & Motivos: & & & \\
\hline & Respeto leyes & 0.01 & 0.02 & 1.02 \\
\hline & Confianza institucional & $-0.04^{* * *}$ & 0.01 & 0.96 \\
\hline & Víctima de delito & $-0.29^{* * *}$ & 0.09 & 0.74 \\
\hline & Capacidades: & & & \\
\hline & Eficacia política & $-0.12^{* * *}$ & 0.02 & 0.88 \\
\hline & Mujer & $0.23^{* * *}$ & 0.07 & 1.27 \\
\hline & Ingreso del hogar & 0.01 & 0.02 & 1.01 \\
\hline & Educación & -0.001 & 0.02 & 1.00 \\
\hline & Desempleado & 0.02 & 0.07 & 1.02 \\
\hline & Edad & -0.0009 & 0.002 & 1.00 \\
\hline & Movilización: & & & \\
\hline & PRI & $-0.81^{* * *}$ & 0.08 & 0.44 \\
\hline & PAN & $-0.62^{* * *}$ & 0.09 & 0.54 \\
\hline & PRD & $-0.57^{* * *}$ & 0.12 & 0.56 \\
\hline & Asociacionismo & $-0.19^{* * *}$ & 0.01 & 0.82 \\
\hline & Voto 2012 & $-0.59^{* * *}$ & 0.10 & 0.55 \\
\hline & Insatisfacción & 0.03 & 0.06 & 1.03 \\
\hline & Indiferencia & $0.15^{*}$ & 0.08 & 1.17 \\
\hline \multirow[t]{20}{*}{ Apático } & Intercepto & $3.78^{* * *}$ & 0.23 & - \\
\hline & Motivos: & & & \\
\hline & Respeto leyes & $0.08^{* * *}$ & 0.02 & 1.09 \\
\hline & Confianza institucional & $-0.04^{* * *}$ & 0.01 & 0.96 \\
\hline & Víctima de delito & $-0.43^{* * *}$ & 0.10 & 0.65 \\
\hline & Capacidades: & & & \\
\hline & Eficacia política & -0.04 & 0.03 & 0.96 \\
\hline & Mujer & 0.04 & 0.07 & 1.05 \\
\hline & Ingreso del hogar & -0.01 & 0.02 & 0.99 \\
\hline & Educación & $-0.14^{* * *}$ & 0.02 & 0.87 \\
\hline & Desempleado & $0.20^{* *}$ & 0.07 & 1.22 \\
\hline & Edad & $-0.008^{* * *}$ & 0.002 & 0.99 \\
\hline & Movilización: & & & \\
\hline & PRI & $-0.56^{* * *}$ & 0.08 & 0.57 \\
\hline & PAN & $-0.52^{* * *}$ & 0.10 & 0.59 \\
\hline & PRD & $-0.35^{* * *}$ & 0.13 & 0.70 \\
\hline & Asociacionismo & $-0.51^{* * *}$ & 0.02 & 0.60 \\
\hline & Voto 2012 & $-0.87^{* * *}$ & 0.10 & 0.42 \\
\hline & Insatisfacción & $-0.15^{* *}$ & 0.07 & 0.86 \\
\hline & Indiferencia & $0.23^{* *}$ & 0.09 & 1.26 \\
\hline
\end{tabular}


Tabla 4. Análisis multinomial para distintos tipos de participación (cont.)

\begin{tabular}{|c|c|c|c|c|}
\hline Tipo $^{\mathrm{a}}$ & & $\beta$ & Error & $\operatorname{Exp}(\beta)$ \\
\hline \multirow[t]{20}{*}{ Hiperactivo } & Intercepto & $-0.69^{*}$ & 0.39 & - \\
\hline & Motivos: & & & \\
\hline & Respeto leyes & -0.07 & 0.05 & 0.93 \\
\hline & Confianza institucional & $-0.03^{* *}$ & 0.01 & 0.96 \\
\hline & Víctima de delito & 0.22 & 0.15 & 1.25 \\
\hline & Capacidades: & & & \\
\hline & Eficacia política & $0.11^{* *}$ & 0.05 & 1.12 \\
\hline & Mujer & -0.10 & 0.13 & 0.90 \\
\hline & Ingreso del hogar & $0.08^{* *}$ & 0.03 & 1.09 \\
\hline & Educación & -0.03 & 0.03 & 0.97 \\
\hline & Desempleado & $0.24^{*}$ & 0.13 & 1.27 \\
\hline & Edad & -0.001 & 0.004 & 1.00 \\
\hline & Movilización: & & & \\
\hline & PRI & $-0.37^{* *}$ & 0.15 & 0.69 \\
\hline & PAN & $-0.39 * *$ & 0.18 & 0.67 \\
\hline & PRD & 0.16 & 0.21 & 1.18 \\
\hline & Asociacionismo & $0.18^{* * *}$ & 0.02 & 1.20 \\
\hline & Voto 2012 & $-0.46^{* * *}$ & 0.18 & 0.63 \\
\hline & Insatisfacción & 0.01 & 0.12 & 1.02 \\
\hline & Indiferencia & $-0.34^{* *}$ & 0.17 & 0.71 \\
\hline$N$ & 8157 & & & \\
\hline
\end{tabular}

${ }^{a}$ Categoría de referencia es Convencional.

Log verosimilitud $=-8668.4$

Prueba de razón de verosimilitud: $\chi^{2}=1511(\mathrm{p}<0.001)$

Códigos de significancia: ${ }^{*} \mathrm{p}<0.1,{ }^{* *} \mathrm{p}<0.05,{ }^{* * *} \mathrm{p}<0.01$

En comparación con el grupo de ciudadanos convencionales, es menos probable que un individuo de la muestra pertenezca al tipo apolítico si reporta mayor confianza en las instituciones, si fue víctima de un delito y si participó (votó) en las elecciones generales. Por el contrario, es más probable que un individuo sea parte del tipo apolítico si es indiferente a la democracia. En términos de capacidades, una mayor eficacia política y ser hombre reduce la probabilidad de que un individuo sea parte de este grupo, en relación con el grupo convencional. En términos de movilización, todos los predictores reducen las razones de momios para el grupo apolítico. En particular, es menos probable que los individuos que se identifican con cualquiera de los tres principales partidos políticos o que son miembros de organizaciones de ciudadanos sean parte del grupo de ciudadanos apolíticos. 
La percepción de que se respetan las leyes mexicanas y la indiferencia hacia la democracia aumentan la probabilidad de que un individuo sea parte del tipo apático. Por el contrario, mayor confianza institucional, haber sido víctima de un delito, haber votado en 2012 y reportarse insatisfecho con la democracia reducen la probabilidad de ser clasificado como apático. Es decir, los ciudadanos insatisfechos, en general, no son apáticos. Además, es menos probable que los más educados o con mayor edad sean apáticos. El ser desempleado aumenta la probabilidad de ser parte del grupo de ciudadanos apáticos. Como en el caso de los ciudadanos apolíticos, contar con opciones de movilización reduce las razones de momios del grupo apático en relación con el convencional.

Finalmente, en comparación con el perfil de los ciudadanos convencionales, es más probable que un individuo de la muestra sea clasificado como hiperactivo si él o ella reportó menor confianza política, no votó en 2012 y si no se declaró indiferente ante la democracia. También es más probable que un individuo sea clasificado en el tipo hiperactivo si cree que puede influir en el gobierno (mayor nivel de eficacia política), cuenta con mayores ingresos o si estaba desempleado al momento de la recolección de datos. Con respecto a las co-variables de movilización, es menos probable que un individuo que se identifica con el PRI o el PAN sea parte del grupo de hiperactivos. Por el contrario, ser miembro de una organización ciudadana incrementa las posibilidades de ser parte de este tipo.

\section{DISCUSIÓN Y CONCLUSIÓN}

Antes de concluir, es importante tratar tres potenciales limitaciones de este estudio. Primero, el estudio se realizó con datos de México. Tal especificidad impone límites a la generalización de nuestra tipología y las observaciones. Por ejemplo, aunque México se asemeja a otros países de la región en términos de participación electoral, como ya se ha dicho, difiere en términos de otras formas de participación política, particularmente en lo que respecta a tasas de asociacionismo (INE 2014). Hasta qué punto esta tipología puede extrapolarse más allá del caso mexicano constituye una pregunta para estudios futuros. No obstante, el análisis del caso mexicano demuestra la utilidad de estudiar conjuntamente la participación en actividades políticas y en actividades comunitarias, y que este diseño puede ofrecer información adicional si se replica en otros contextos.

Segundo, el estudio usó medidas retrospectivas. Esto significa que utilizamos datos sobre participación autorreportados, en lugar de fuentes más objetivas como archivos o registros oficiales. Aunque el diseño tiene ventajas importantes — como la replicabilidad y la eficiencia—, también puede estar sujeto a sesgos de deseabilidad social. Las medidas objetivas de participación (distintas del voto) son difíciles de obtener en algunos casos (por ejemplo, membresía en partidos políticos o cartas enviadas a políticos) y casi imposibles en otras (por ejemplo, ayudar a un extraño). Sin embargo, futuras investigaciones ciertamen- 
te podrían beneficiarse de múltiples fuentes de información que combinen mediciones de registro con datos de encuestas.

Tercero, nuestro análisis de tipos de participación se hizo con datos individuales de encuesta, empero, sin tomar en cuenta la contextualización geográfica (regional) de las personas. Dado que existe evidencia de variaciones en la calidad de las instituciones democráticas en distintas partes de México (cf. IDD-Mex 2018), ${ }^{9}$ se requiere más investigación para comprender los vínculos entre las diferentes formas de participación y el desarrollo democrático de las regiones. Análisis preliminares en nuestra muestra y que podrían ser la base para estudios futuros no solo sugieren que existe una relación entre el tipo de participación y la ubicación geográfica de los individuos, sino que también los estados (provincias) mexicanos con distintos niveles de desarrollo democrático difieren en su propensión a diferentes formas de participación política y comunitaria. Más aún, dada una posible relación entre la frecuencia de distintos perfiles de participación y el contexto, otra posible avenida es el estudio comparado entre países. Como observamos al principio, México se asemeja en términos de participación a otras democracias. De modo que sería interesante estudiar las posibles similitudes en términos de nuestra tipología en distintas democracias y evaluar si las diferencias, tanto en términos de perfiles de participación, cuanto en lo que toca a las posibles correlaciones con la calidad de las instituciones democráticas pueden compararse con otros casos.

A pesar de las limitaciones, el presente análisis de patrones de participación demuestra que nuestra tipología tiene validez empírica y que, además, es informativa. La evidencia recolectada en este estudio sugiere que los ciudadanos pueden diferir en sus patrones de participación política, así como su involucramiento en actividades comunitarias. Esto es importante porque la falta de participación política no necesariamente debe interpretarse como apatía. Como demuestra el análisis, una porción considerable de la muestra de ciudadanos mexicanos está formada por individuos apolíticos que participan mediante actividades comunitarias y que evitan involucrarse en formas convencionales de participación política o actividades políticas de protesta. De hecho, los ciudadanos apolíticos formaron el mayor subgrupo en la muestra estudiada $(45 \%)$ y la participación comunitaria fue más frecuente que otras formas de participación. En la medida en que los ciudadanos apolíticos se involucran en formas de participación comunitaria permanecen activos. Esto implica que hay ciudadanos que pueden seguir participando cívicamente fuera de los canales e instancias institucionales. Sin embargo, es importante anotar que esta forma de participación ciudadana puede estar correlacionada (como en el caso de la muestra estudiada) con mayor una indiferencia hacia la democracia. 
Por otro lado, diferencias en motivaciones, capacidades y oportunidades de movilización son importantes para comprender la tipología de participación. La evidencia sugiere que, en comparación con el perfil de ciudadanos convencionales, las diferentes categorías "alternativas" difieren en sus antecedentes típicos. En este sentido, los resultados confirman en buena medida las predicciones de los modelos de movilización en tanto las personas con acceso a redes o grupos o que se identifican con organizaciones políticas tienden a participar más. Las predicciones del modelo socioeconómico son menos consistentes en nuestros datos; sin embargo, en general, personas con diferentes dotaciones de recursos tangibles o intangibles sí tienden a tener diferentes patrones de participación ciudadana. Algunas variables se correlacionan con todos los tipos de la tipología. Por ejemplo, la victimización delictiva (cf. Barahona y Rivas 2014), la identificación con partidos políticos, la membresía en organizaciones cívicas (cf. Klesner 2009) y el voto son aparentemente "catalizadores políticos" poderosos (es decir, reducen la probabilidad de una ciudadanía apática o apolítica). Las diferencias también son interesantes. Por ejemplo, una mayor eficacia política parece reducir la probabilidad de una ciudadanía apolítica y, a su vez, aumentar la "hiperactividad". La creencia de que se respeta la ley aumenta la probabilidad del tipo apático. Sin embargo, el aumento de la confianza en las instituciones reduce el factor de riesgo relativo del tipo apolítico, apático e hiperactivo, en relación con el tipo convencional.

La evidencia también respalda la idea de que la insatisfacción con la política y la indiferencia con la democracia tienen efectos diferenciados. Creer que la democracia únicamente beneficia a pocas personas reduce la probabilidad de una ciudadanía apática (es decir, esta insatisfacción parece activar a los ciudadanos). Ser indiferente hacia la democracia, por el contrario, aumenta la probabilidad tanto del tipo apolítico como del apático y reduce la del tipo de hiperactivo. Por tanto, parece que los ciudadanos indiferentes se alejan de la política, pero al menos algunos se decantan por la participación comunitaria (cf. Yoo 2010).

Por tanto, se puede concluir que los diferentes perfiles de participación ciudadana están relacionados con conjuntos específicos de co-variables en términos de capacidades, motivaciones y oportunidades de movilización. Además, los resultados sugieren que la insatisfacción y la indiferencia, como mecanismos diferentes, podrían tener efectos en la participación o falta de ésta, dentro y fuera de la esfera política. Como ya argumentó Di Palma (1970), la comprensión de las diferentes razones por las que los ciudadanos se vuelcan hacia la participación o se alejan de ella sigue siendo un tema relevante para los estudiosos de la gobernanza democrática. No todas las formas de participación o, para el caso, de falta de participación política son iguales y las diferencias merecen ser estudiadas con mayor consideración. 


\section{REFERENCIAS}

Adler, Richard P. y Judy Goggin. 2005. "What Do We Mean by "Civic Engagement"? Journal of Transformative Education 3(3): 236-253.

Aldenderfer, Mark S. y Roger K. Blashfield. 1984. Cluster Analysis. Newbury Park: Sage.

Almond, Gabriel y Sidney Verba. 1963. The Civic Culture. Political Attitudes and Democracy in Five Nations. Princeton: Princeton University Press.

Ayala, Louis J. 2000. "Trained for Democracy: The Differing Effects of Voluntary and Involuntary Organizations on Political Participation". Political Research Quarterly 53(1): 99115.

Barahona, Elena M. y Cristina Rivas. 2014. "'Estamos hasta la madre'. Making Victims Visible in Mexico's Current 'Crime Epidemic'". En Democracy in Mexico, editado por Salvador Martí i Puig, Reynaldo Y. Ortega Ortiz, Fernanda Somuano Ventura y Claire Wright. Londres: University of London, 219-44.

Bateson, Regina. 2012. "Crime Victimization and Political Participation". American Political Science Review 106(3): 570-87.

Béjar, Alejandro. 2015. "Global Economic Crisis and Social Movements in Mexico and North America." Social Justice 42(142): 237-251.

Bensusán, Graciela y Kevin Middlebrook. 2013. Sindicatos y Política en México: Cambios, Continuidades y Contradicciones. México: Flacso México-UAM Xochimilco.

Bilder, Christopher R. y Thomas M. Loughin. 2015. Analysis of Categorical Data with R. Boca Raton: CRC Press.

Booth, John A. y Mitchell A. Seligson. 1978. Political Participation in Latin America. Nueva York: Holmes y Meier.

Brady, Henry E. 1999. "Political Participation". En Measures of Political Attitudes, editado por John P. Robinson, Phillip R. Shaver y Lawrence S. Wrightsman, San Diego: Academic Press, 737-801.

Brady, Henry E., Sidney Verba y Kay Lehman Schlozman. 1995. “Beyond SES: A Resource Model of Political Participation". American Political Science Review 89(2): 271-94.

Brooks, Sarah M. 2014. "Insecure Democracy: Risk and Political Participation in Brazil”. Journal of Politics 76(4): 972-85.

Campbell, David E. 2006. Why We Vote? How Schools and Communities Shape Our Civic Life. Princeton: Princeton University Press.

Castillo, Juan C., Diego Palacios, Alfredo Joignant y Maximiliano Tham. 2015. “Inequality, Distributive Justice and Political Participation: An Analysis of the Case of Chile". Bulletin of Latin American Research 34(4): 486-502.

Dalton, Russell J. 2008. Citizen Politics. Public Opinion and Political Parties in Advanced Industrial Democracies. Washington, DC: CQ Press.

Davis, Diane E. 1991. “Urban Social Movements. Intrastate Conflicts over Urban Policy, and Political Change in Contemporary Mexico." En Breaking Chains: Social Movements and Collective Action, editado por Michael Peter Smith. New Brunswick: Transaction, 133-163.

De Weerd, Marga, Mireille Gemmeke, Josine Rigter y Coen Van Rij. 2005. Indicators for Monitoring Active Citizenship and Citizenship Education. Amsterdam: Regioplan Beleidsonderzoek.

Di Palma, Giuseppe. 1970. Apathy and Participation. Mass Politics in Western Societies. Nueva York: The Free Press.

Dunn, John C. 1973. "A Fuzzy Relative of the ISODATA Process and its Use in Detecting Compact Well-Separated Clusters". Journal of Cybernetics 3(3): 32-57.

Ekman, Joakim y Erik Amnå. 2012. "Political Participation and Civic Engagement: Towards a New Typology". Human Affairs 22(3): 283-300.

Escobar, Arturo y Sonia E. Álvarez. 1992. The Making of Social Movements in Latin America: Identity, Strategy, and Democracy. San Francisco: Westview. 
Fennema, Meindert y Jean Tillie. 1999. "Political Participation and Political Trust in Amsterdam: Civic Communities and Ethnic Networks". Journal of Ethnic and Migration Studies 25(4): 703-26.

Fiorina, Morris. 1999. "Extreme Voices: A Dark Side of Civic Engagement". En Civic Engagement in American Democracy, editado por Theda Skocpol y Morris Fiorina. Washington: Brookings Institution Press, 395-425.

Hay, Colin. 2007. Why We Hate Politics. Cambridge: Polity.

Heywood, Andrew. 2000. Key Concepts in Politics. Nueva York: St. Martin's Press.

Inclán, María. 2018. The Zapatista Movement and Mexico's Democratic Transition. Oxford: Oxford University Press.

IDD-Mex 2018. Índice de Desarrollo Democrático de México. Recuperado el 13 de enero de 2019 de http:/ /idd-mex.org/2018/informes/2018/index.html

INE. 2014. Informe país sobre la calidad de la ciudadanía en México. Ciudad de México: INE.

Isin, Engin F. y Patricia K. Wood. 1999. Citizenship and Identity. Londres: Sage.

Johnson, April A. 2014. "Ambivalence, Political Engagement and Context”. Political Studies 62(3): 502-21.

Kam, Cindy D. 2012. "Risk Attitudes and Political Participation". American Journal of Political Science 56(4): 817-36.

Klesner, Joseph L. 2009. "Who Participates? Determinants of Political Action in Mexico". Latin American Politics and Society 51(2): 59-90.

Lander, Jared P. 2014. R for Everyone. Upper Saddle River: Adison-Wesley.

Lehman, Kay, Sidney Verba y Henry E. Brady. 2012. Unequal Political Voice and the Broken Promise of American Democracy. Princeton: Princeton University Press.

Leighley, Jan E. 1995. “Opportunities and Incentives: A Field Essay on Political Participation". Political Research Quarterly 48(1): 181-209.

Lindenberg, Siegwart. 2013. "Cognition and Governance: Why Incentives Have to Take a Back Seat". En Handbook of economic organization. Integrating economic and organization theory, editado por Anna Grandori. Cheltenham: Edward Elgar, 41-61.

Macedo, Stephen, Yvette Alex-Assensoh, Jeffrey M. Berry, Michael Brintnall, David E. Campbell, Luis Ricardo Fraga, Archon Fung, William A. Galston, Christopher F. Karpowitz, Margaret Levi, Meira Levinson, Keena Lipsitz, Richard G. Niemi, Robert D. Putnam, Wendy M. Rahn, Rob Reich, Robert R. Rodgers y Todd Swanstrom. 2005. Democracy at Risk: How Political Choices Undermine Citizen Participation, and What We Can Do About It. Washington: Brookings Institution.

Marshall, Thomas H. 1950. Citizenship and Social Class. Cambridge: Cambridge University Press.

Martí i Puig, Salvador e Iván Llamazares. 2011. "La protesta política. ¿Quiénes movilizan y por qué lo hacen?" en La democracia en México. Un análisis a 10 años de la alternancia, editado por Salvador Martí i Puig, Reynaldo Y. Ortega y Fernanda Somuano. Barcelona: Bellaterra, 69-95.

McClurg, Scott D. 2003. "Social Networks and Political Participation: The Role of Social Interaction in Explaining Political Participation". Political Research Quarterly 56(4): 449-64.

Milbrath, Lester W. y M. Lal Goel. 1977. Political Participation: How and Why Do People Get Involved in Politics? Chicago: Rand McNally.

Mill, John Stuart. 1977. Considerations on Representative Government. Londres: M. Dent and Son.

Nickerson, David, W. 2014. "Do Voter Registration Drives Increase Participation. For Whom and When?" The Journal of Politics 77(1): 88-101.

Norris, Pipa. 1999. Critical Citizens. Global Support for Democratic Governance. Nueva York: Oxford University Press.

Norris, Pipa. 2002. Democratic Phoenix. Reinventing Political Activism. Cambridge: Cambridge University Press.

O'Toole, Therese, David Marsh y Su Jones. 2003. "Political Literacy Cuts Both Ways: The Politics of Non-Participation among Young People". Political Quarterly 74(3): 349-60. 
Pateman, Carole. 1970. Participation and Democratic Theory. Cambridge: Cambridge University Press.

Putnam, Robert. 1995. “Bowling Alone. America's Declining Social Capital". Journal of Democracy 6(1): 65-78.

Putnam, Robert. 2000. Bowling Alone. The Collapse and Revival of American Community. Nueva York: Simon y Schuster.

Quintelier, Ellen, y Jan W. Van Deth. 2014. "Supporting Democracy: Political Participation and Political Attitudes. Exploring Causality Using Panel Data". Political Studies 62(S1): 153-71.

Ramírez, Miguel Ángel. 2016. Movimientos Sociales en México: Apuntes Teóricos y Estudios de Caso. México: UAM.

Rosenstone, Steven y John Mark Hansen. 1993. Mobilization, Participation and Democracy in America. Nueva York: Macmillan.

Rousseau, Jean Jacques. 1973. The Social Contract. Londres: M. Dent and Son.

Skocpol, Theda, y Morris P. Fiorina. 1999. Civic Engagement in American Democracy. Washington: Brookings Institution Press.

Stoker, Gerry. 2006. Why Politics Matters: Making Democracy Work. Basingstoke: Palgrave Macmillan.

Tarrow, Sidney. 1998. Power in movement. Cambridge: Cambridge University Press.

Teorell, Jan. 2006. "Political Participation and Three Theories of Democracy: A Research Inventory and Agenda". European Journal of Political Research 45(5): 787-810.

Tilly, Charles. 1978. From Mobilization to Revolution. Reading: Addison-Wesley.

Van Nierop, Petra. 2006. Study on Active Citizenship Education. Bruselas: GHK.

Verba, Sidney, Norman H. Nie y Jae On Kim. 1978. Participation and Political Equality. Nueva York: Cambridge University Press.

Verba, Sidney, Kay Lehman Schlozman y Henry E. Brady. 1995. Voice and Equality: Civic Voluntarism in American Politics. Cambridge: Harvard University Press.

Wattenberg, Martin. 2002. Where Have All the Voters Gone? Cambridge: Harvard University Press.

Weiner, Myron. 1971. "Political Participation: Crisis of the Political Process". En Crisis and Sequences in Political Development, editado por Leonard Binder y Joseph La Palombara. Princeton: Princeton University Press, 159-204.

Yoo, Sung-jin. 2010. “Two Types of Neutrality: Ambivalence Versus Indifference and Political Participation". The Journal of Politics 72(1): 163-77.

Recibido: 19 de junio de 2018

Aceptado: 30 de octubre de 2019

Fernando Nieto: Profesor-investigador en el Centro de Estudios Internacionales de El Colegio de México. Es doctor en sociología y maestro en ciencias del comportamiento por la Universidad de Groninga, Países Bajos. Sus líneas de investigación y especialización incluyen organización y confianza en el gobierno, corrupción y patologías burocráticas y la profesionalización de las organizaciones gubernamentales. Es miembro del Sistema Nacional de Investigadores de México (nivel I). En 2016, obtuvo el Premio Erasmo a la investigación en ciencias sociales de la Real Academia Neerlandesa de Ciencias. Correo electrónico: fnieto@colmex.mx

Fernanda Somuano: Doctora en ciencia política por la Universidad de Iowa y maestra en administración y políticas públicas por la London School of Economics and Political Science. Es profesora-investigadora del Centro de Estudios Internacionales de El Colegio de México desde enero del 2001. Es especialista y ha publicado diversos artículos y libros en temas de democratización, opinión pública, sociedad civil y ciudadanía en México. Es miembro del Sistema Nacional de Investigadores de México (nivel III) y forma parte del Comité Editorial de la Revista Foro Internacional y del Fondo de Cultura Económica. Correo electrónico: fsomuano@colmex.mx 